\title{
Multifunctional Roles of Plant Dehydrins in Response to Environmental Stresses
}

\author{
Yang Liu, Qiping Song, Daxing Li, Xinghong Yang * and Dequan Li* \\ State Key Laboratory of Crop Biology, Shandong Key Laboratory of Crop Biology, College of Life Sciences, Shandong \\ Agricultural University, Tai'an, China
}

OPEN ACCESS

Edited by:

Sergio J. Ochatt,

INRA - UMR 1347 Agroécologie,

France

Reviewed by:

Fisun G. Çelikel,

Ondokuz Mayıs University, Turkey

Khawar Jabran,

Duzce University, Turkey

*Correspondence:

Dequan Li

dqli@sdau.edu.cn

Xinghong Yang

xhyang@sdau.edu.cn

Specialty section:

This article was submitted to Crop Science and Horticulture,

a section of the journal

Frontiers in Plant Science

Received: 14 March 2017

Accepted: 29 May 2017

Published: 09 June 2017

Citation:

Liu Y, Song $Q$, Li D, Yang X and Li D (2017) Multifunctional Roles of Plant Dehydrins in Response

to Environmental Stresses.

Front. Plant Sci. 8:1018.

doi: 10.3389/fpls.2017.01018
To respond to environmental changes, plants have developed complex mechanisms that allow them to rapidly perceive and respond to abiotic stresses. Late embryogenesis abundant (LEA) proteins are a large and diverse family that play important roles in environmental stress tolerance in plants. Dehydrins belong to group II LEA proteins, which are considered stress proteins involved in the formation of plants' protective reactions to dehydration. Some studies have demonstrated that dehydrins could binding metal ions or lipid vesicles. In vitro experiments revealed that dehydrins could protect the activity of enzyme from damage caused by environmental stress. Although many studies have been conducted to understand their roles in abiotic stresses, the molecular function of dehydrins is still unclear. In this review, to generate new ideas for elucidating dehydrins' functions, we highlight the functional characteristics of dehydrins to understand their roles under environmental stress in plants.

Keywords: dehydrin, conserved segments, LEA protein, abiotic stresses, function

\section{INTRODUCTION}

Late embryogenesis abundant (LEA) proteins are related with the acquisition of desiccation tolerance in plants and are accumulated during the late stages of seed development (Dure and Galau, 1981; Liu et al., 2013, 2016). According to the conserved segments, LEA proteins can be categorized into seven different groups (Battaglia et al., 2008). Dehydrins belong to the group II LEA proteins, which were initially identified as the " $\mathrm{D}-11$ " family in the developing cotton embryos. Dehydrins are considered stress proteins involved in the formation of plants' protective reactions to dehydration. They can also be considered as hydrophilins. They are highly hydrophilic, containing a high proportion of charged and polar amino acids and a low fraction of hydrophobic, non-polar residues, and lack tryptophan and cysteine residues. Based on the presence of conserved sequences (Y-, S-, and K-segments), dehydrins were classified into the different sub-classes YnKn, YnSKn, KnS, SKn and Kn (Garay-Arroyo et al., 2000).

Although the function of dehydrins has not been clearly understood, many studies have demonstrated dehydrins play important roles in abiotic stress tolerance. Overexpression of the Prunus mume dehydrin PmLEAs in tobacco and Escherichia coli enhances tolerance to cold and drought (Bao et al., 2017). Overexpression of the Solanum habrochaites dehydrins ShDHN enhances transgenic tomato tolerance to multiple abiotic stresses (Liu et al., 2015). Most dehydrins are distributed along with several ubiquitous dehydration-stress response protein types in plants (Bao et al., 2017; Fan et al., 2017). However, some dehydrins were also found distributed in the vegetative tissues of plants grown under normal conditions, which suggested that the 
dehydrins may play important roles in the plant growth. Recently, studies indicate that the Medicago truncatula $\mathrm{Y}_{2} \mathrm{~K}_{4}$-type dehydrin MtCAS31 could interact with AtICE1 (an inducer of CBF expression 1). The interaction of AtICE1with MtCAS31 plays important roles in stomatal development, enhancing the drought resistance by decreasing in stomatal density of transgenic Arabidopsis thaliana (Xie et al., 2012).

\section{THE ROLES OF THE DIFFERENT CONSERVED SEGMENTS IN DEHYDRINS}

The K-segment can form an amphiphilic $\alpha$-helix, which representing a highly conserved 15 amino acid segment (EKKGIMDKIKEKLPG) that has been found in all dehydrins (Malik et al., 2017). The $\alpha$-helix of dehydrins may help stabilize the proteins and the cellular membranes under environmental stress conditions. The Citrus unshiu $\mathrm{K}_{3} \mathrm{~S}$-type dehydrin CuCOR19 was found to form an $\alpha$-helix in the presence of SDS (sodium dodecyl sulfate) (Hara et al., 2001). The K-segment of the maize dehydrin DHN1 is essential for binding to the anionic phospholipid vesicles, and the adoption of $\alpha$-helicity by the K-segment accounts for the conformational change in dehydrins upon binding to SDS or anionic phospholipid vesicles (Koag et al., 2009). The $\mathrm{K}$-segment of wheat dehydrin $\mathrm{DHN}-5$ is required for the protection of $\beta$-glucosidase and LDH (lactate dehydrogenase) activities in vitro (Drira et al., 2013). The lysine-rich segment of the Craterostigma plantagineum disordered stress protein CDeT11-24 is important for the protection of proteins from damage caused by water stress and the phosphatidic acid (PA) binding (Petersen et al., 2012). The cold-induced dehydrin Lti30 can bind to cellular membranes via its K-segments, which locally fold into $\alpha$-helix on the cellular membrane surface. The protein Lti30's K-segments electrostatically trap the negatively charged lipid head (Eriksson et al., 2011, 2016).

The Y-segment, representing a conserved segment $[\mathrm{V} / \mathrm{T}] \mathrm{D}[\mathrm{E} / \mathrm{Q}] \mathrm{YGNP}$, is usually found in various tandem copies in the N-terminus of dehydrins (Campbell and Close, 1997). The Y-segment of dehydrins has sequence similarity to the nucleotide-binding site of plant and bacterial chaperones [(V/T) DEYGNP]. However, there is no experimental evidence suggesting that the Y-segment binds nucleotides (Close, 1996; Hughes et al., 2013).

Some dehydrins contain the S-segment, which consists of a tract of Ser residues and can be phosphorylated. The phosphorylation of the S-segment is suggested to promote dehydrins interaction with the specific signal peptides followed by their translocation into the nucleus (Goday et al., 1994; Close, 1996; Jiang and Wang, 2004). The S-segment can be phosphorylated by protein kinase, which may influence the location and the ability to bind metal ions (Goday et al., 1994; Alsheikh et al., 2003). The three amino acid residues EDD of the maize dehydrin Rab17 are the CKII phosphorylation recognition site (Jensen et al., 1998).
The $\Phi$-segments are less conserved motifs, which lay interspersed between K-segments (Campbell and Close, 1997).

\section{THE FUNCTION OF DEHYDRINS}

\section{Binding Metal lons}

Under control conditions, catalytic metal ions (such as $\mathrm{Cu}^{2+}$ and $\mathrm{Zn}^{2+}$ ) mainly exist as metal-protein complexes in plants. Nevertheless, they can be released as free ions when plants are subjected to stress conditions. These free ions are required to produce ROS (reactive oxygen species) via the Fenton or the Haber-Weiss reactions (Mittler, 2002). It has been reported that the Ricinus KS-type dehydrin ITP is the first member of the LEA protein family shown to be active in the long-distance transport micronutrients (Krüger et al., 2002). The Arabidopsis thaliana dehydrin AtHIRD11 and citrus dehydrin CuCOR15 can bind the metal ions $\mathrm{Fe}^{3+}, \mathrm{Co}^{2+}, \mathrm{Ni}^{2+}, \mathrm{Cu}^{2+}$ and $\mathrm{Zn}^{2+}$ over $\mathrm{Mg}^{2+}$ and $\mathrm{Ca}^{2+}$ (Hara et al., 2005, 2011).

\section{Binding DNA}

The bioinformatic analysis of the "Protein or Oligonucleotide Probability Profile (POPP)" predicted that dehydrins could bind DNA (Wise and Tunnacliffe, 2004). Until now, several studies have reported that LEA protein can bind DNA. Citrus dehydrin CuCOR15 can bind nucleic acids, a process that depends on zinc ions. The histidine-rich domain (TTDVHHQQQYHGGEH) and the lysine-rich domain (GGEGAHGEEKKKKKKEKKK) were the DNA-binding domains (Hara et al., 2009). $\mathrm{Y}_{2} \mathrm{~K}$-type dehydrin VrDhn1 exhibited a low affinity for non-specific interaction with DNA, and the exogenous addition metal ions of $\mathrm{Zn}^{2+}$ or $\mathrm{Ni}^{2+}$ stimulated the interaction (Lin et al., 2012).

\section{Binding Phospholipid}

Phosphatidic acid is the important stress-signaling phospholipid, and its accumulation is triggered in response to various environmental stress conditions, such as salinity, drought and cold (Frank et al., 2000; Katagiri et al., 2005). The PA concentration in the turgescent plant membranes is only approximately $1 \%$, but increases under water deficit stress. The formation of PA depends on the activity of phospholipase D, which can be induced by ABA and dehydration (Katagiri et al., 2001).

Many studies have demonstrated that dehydrin can bind with phospholipids, and the binding was not limited to a certain type of phospholipids (Kooijman et al., 2007; Koag et al., 2009; Petersen et al., 2012). The maize $\mathrm{SK}_{2}$ - type dehydrin DHN1 can bind PA. Although most of the DHN1 protein is disorder, the K-segment can adopt $\alpha$-helical conformation. An electrostatic nature of the interaction has been postulated. The basic amino acids, such as arginine and lysine, are required for the interaction in the binding domain (Kooijman et al., 2007; Koag et al., 2009). The Kn-type dehydrin Lti30 can interact electrostatically with vesicles of both negatively charged phospholipids (phosphatidic acid, phosphatidyl serine and phosphatidyl glycerol) and zwitterionic (phosphatidyl choline). The Lti30 interaction of lipid is regulated by phosphorylation, 
$\mathrm{pH}$ dependent His on/off switch, and the reversal of membrane binding by proteolytic digest (Eriksson et al., 2011).

\section{PROTECTING THE ACTIVITY OF PROTEINS}

The hypothesis that dehydrin can bind proteins to prevent its denaturation has been suggested for many years. Many studies have demonstrated that dehydrin can protect the activity of $\mathrm{LDH}$ and malate dehydrogenase from damage caused by various environmental stresses (Drira et al., 2013, 2015; Yang et al., 2015). The KS-type dehydrin AtHIRD11 recovers LDH activity inhibited by copper with the contribution of histidine residues (Hara et al., 2016). However, the interaction between the dehydrins and the protected proteins has been not found.

\section{SCAVENGING THE REACTIVE OXYGEN SPECIES}

Many studies have indicated that dehydrins can directly scavenge the free radicals. The citrus dehydrin CuCOR19 displays a stronger inhibition against free radical-inducing peroxidation. The overexpression CuCOR19 in transgenic tobacco can inhibit lipid peroxidation under cold stress (Hara et al., 2003). The amino acids, such as histidine, lysine and glycine, are targets for radical-mediated oxidation (Dean et al., 1997). The content of the three amino acids is high in mostly dehydrins, which can reduce the level of ROS. The Arabidopsis thaliana KS-type dehydrin AtHIRD11 can reduce ROS generation from copper. The length of the peptides and the histidine contents are fundamental factors that can influence

\section{REFERENCES}

Alsheikh, M. K., Heyen, B. J., and Randall, S. K. (2003). Ion binding properties of the dehydrin ERD14 are dependent upon phosphorylation. J. Biol. Chem. 278, 40882-40889. doi: 10.1074/jbc.M307151200

Bao, F., Du, D., An, Y., Yang, W., Wang, J., Cheng, T., et al. (2017). Overexpression of Prunus mume dehydrin genes in tobacco enhances tolerance to cold and drought. Front. Plant Sci. 8:151. doi: 10.3389/fpls.2017.00151

Battaglia, M., Olvera-Carrillo, Y., Garciarrubio, A., Campos, F., and Covarrubias, A. A. (2008). The enigmatic LEA proteins and other hydrophilins. Plant Physiol. 148, 6-24. doi: 10.1104/pp.108.120725

Campbell, S. A., and Close, T. J. (1997). Dehydrins: genes, proteins, and associations with phenotypic traits. New Phytol. 137, 61-74. doi: 10.1046/j. 1469-8137.1997.00831.x

Close, T. J. (1996). Dehydrins: emergence of a biochemical role of a family of plant dehydration proteins. Physiol. Plant. 97, 795-803. doi: 10.1111/j.13993054.1996.tb00546.x

Dean, R. T., Fu, S., Stocker, R., and Davies, M. J. (1997). Biochemistry and pathology of radical-mediated protein oxidation. Biochem. J. 324, 1-18. doi: 10.1042/bj3240001

Drira, M., Saibi, W., Amara, I., Masmoudi, K., Hanin, M., and Brini, F. (2015). Wheat dehydrin K-segments ensure bacterial stress tolerance, antiaggregation and antimicrobial effects. Appl. Biochem. Biotechnol. 175, 3310-3321. doi: $10.3389 /$ fpls.2015.00406

Drira, M., Saibi, W., Brini, F., Gargouri, A., Masmoudi, K., and Hanin, M. (2013). The K-segments of the wheat dehydrin DHN-5 are essential for the protection the ROS decrease by the KS-type dehydrins (Hara et al., 2013, 2016).

\section{CONCLUSION}

To summarize the data presented above, the functional mechanisms are listed as follows.

(I) Dehydrins can bind metal ions, which can inhibit the production of ROS at the source.

(II) The high content of the antioxidant amino acids, such as lysine, histidine and glycine, can scavenge ROS through oxidative modification.

(III) Dehydrins can non-specifically bind proteins and membranes, which can protect the function and structure of the protein or membrane from damage caused by environmental stresses.

(IV) Dehydrins can bind DNA, which may repair or protect the DNA from damage caused by environmental stresses.

\section{AUTHOR CONTRIBUTIONS}

YL wrote the article. QS and DaL gave positive suggestion about this article. DeL and XY revised the paper and gave positive suggestion. All authors read and approved the manuscript.

\section{ACKNOWLEDGMENTS}

This work was supported by the Shandong Province Natural Science Foundation (ZR2016CQ34) and the Grants from the Nation Natural Science Foundation of China (Nos. 31271633).

of lactate dehydrogenase and $\beta$-glucosidase activities in vitro. Mol. Biotechnol. 54, 643-650. doi: 10.1007/s12033-012-9606-8

Dure, L., and Galau, G. A. (1981). Developmental biochemistry of cotton seed embryogenesis and germination XIII. Regulation of biosynthesis of principal storage proteins. Plant Physiol. 68, 187-194. doi: 10.1104/pp.68. 1.187

Eriksson, S., Eremina, N., Barth, A., Danielsson, J., and Harryson, P. (2016). Membrane-induced folding of the plant-stress protein Lti30. Plant Physiol. 71, 932-943. doi: 10.1104/pp.15.01531

Eriksson, S. K., Kutzer, M., Procek, J., Grobner, G., and Harryson, P. (2011). Tunable membrane binding of the intrinsically disordered dehydrin Lti30, a cold-induced plant stress protein. Plant Cell 23, 2391-2404. doi: 10.1105/tpc. 111.085183

Fan, N., Lv, A., Xie, J., Yuan, S., An, Y., and Zhou, P. (2017). Expression of CdDHN4, a novel YSK2-type dehydrin gene from bermudagrass, responses to drought stress through ABA-dependent signal pathway. Front. Plant Sci. 8:748. doi: $10.3389 /$ fpls.2017.00748

Frank, W., Munnik, T., Kerkmann, K., Salamini, F., and Bartels, D. (2000). Water deficit triggers phospholipase $\mathrm{D}$ activity in the resurrection plant Craterostigma plantagineum. Plant Cell 12, 111-123. doi: 10.1105/tpc.12.1.111

Garay-Arroyo, A., Colmenero-Flores, J. M., Garciarrubio, A., and Covarrubias, A. A. (2000). Highly hydrophilic proteins in prokaryotes and eukaryotes are common during conditions of water deficit. J. Biol. Chem. 275, 5668-5674. doi: $10.1074 /$ jbc. 275.8 .5668

Goday, A., Jensen, A. B., Culiáñez-Macià, F., Albà, M. M., Figueras, M., Serratosa, J., et al. (1994). The maize abscisic-acid responsive protein Rab17 is 
located in the nucleus and interacts with nuclear localization signals. Plant Cell 6, 351-360. doi: 10.1105/tpc.6.3.351

Hara, M., Fujinaga, M., and Kuboi, T. (2005). Metal binding by citrus dehydrin with histidine-rich domains. J. Exp. Bot. 56, 2695-2703. doi: 10.1093/jxb/eri262

Hara, M., Kondo, M., and Kato, T. (2013). A KS-type dehydrin and its related domains reduce $\mathrm{Cu}$-promoted radical generation and the histidine residues contribute to the radical-reducing activities. J. Exp. Bot. 64, 1615-1624. doi: 10.1093/jxb/ert016

Hara, M., Monna, S., Murata, T., Nakano, T., Amano, S., Nachbar, M., et al. (2016). The Arabidopsis KS-type dehydrin recovers lactate dehydrogenase activity inhibited by copper with the contribution of his residues. Plant Sci. 245, 135-142. doi: 10.1016/j.plantsci.2016.02.006

Hara, M., Shinoda, Y., Kubo, M., Kashima, D., Takahashi, I., Kato, T., et al. (2011). Biochemical characterization of the Arabidopsis KS-type dehydrin protein, whose gene expression is constitutively abundant rather than stress dependent. Acta Physiol. Plant. 33, 2103-2116. doi: 10.1007/s11738-011-0749-1

Hara, M., Shinoda, Y., Tanaka, Y., and Kuboi, T. (2009). DNA binding of citrus dehydrin promoted by zinc ion. Plant Cell Environ. 32, 532-541. doi: 10.1111/j. 1365-3040.2009.01947.x

Hara, M., Terashima, S., Fukaya, T., and Kuboi, T. (2003). Enhancement of cold tolerance and inhibition of lipid peroxidation by citrus dehydrin in transgenic tobacco. Planta 217, 290-298. doi: 10.1007/s00425-003-0986-7

Hara, M., Terashima, S., and Kuboi, T. (2001). Characterization and cryoprotective activity of cold-responsive dehydrin from Citrus unshiu. J. Plant Physiol. 158, 1333-1339. doi: 10.1078/0176-1617-00600

Hughes, S. L., Schart, V., Malcolmson, J., Hogarth, K. A., Martynowicz, D. M., Tralman-Baker, E., et al. (2013). The importance of size and disorder in the cryoprotective effects of dehydrins. Plant Physiol. 163, 1376-1386. doi: 10.1104/ pp.113.226803

Jensen, A. B., Goday, A., Figueras, M., Jessop, A. C., and Pages, M. (1998). Phosphorylation mediates the nuclear targeting of the maize Rab17 protein. Plant J. 13, 691-697. doi: 10.1046/j.1365-313X.1998.00069.x

Jiang, X., and Wang, Y. (2004). $\beta$-Elimination coupled with tandem mass spectrometry for the identification of in vivo and in vitro phosphorylation sites in maize dehydrin DHN1 protein. Biochemistry 43, 15567-15576. doi: 10.1021/bi0483965

Katagiri, T., Ishiyama, K., Kato, T., Tabata, S., Kobayashi, M., and Shinozaki, K. (2005). An important role of phosphatidic acid in ABA signaling during germination in Arabidopsis thaliana. Plant J. 43, 107-117. doi: 10.1111/j.1365313X.2005.02431.x

Katagiri, T., Takahashi, S., and Shinozaki, K. (2001). Involvement of a novel Arabidopsis phospholipase D, AtPLD $\delta$, in dehydration-inducible accumulation of phosphatidic acid in stress signalling. Plant J. 26, 595-605. doi: 10.1046/j. 1365-313x.2001.01060.x

Koag, M. C., Wilkens, S., Fenton, R. D., Resnik, J., Vo, E., and Close, T. J. (2009). The K-segment of maize DHN1 mediates binding to anionic phospholipid vesicles and concomitant structural changes. Plant Physiol. 150, 1503-1514. doi: $10.1104 /$ pp.109.136697

Kooijman, E. E., Tieleman, D. P., Testerink, C., Munnik, T., Rijkers, D. T., Burger, K. N., et al. (2007). An electrostatic/hydrogen bond switch as the basis for the specific interaction of phosphatidic acid with proteins. J. Biol. Chem. 282, 11356-11364. doi: 10.1074/jbc.M609737200

Krüger, C., Berkowitz, O., Stephan, U. W., and Hell, R. (2002). A metalbinding member of the late embryogenesis abundant protein family transports iron in the phloem of Ricinus communis L. J. Biol. Chem. 277, 25062-25069. doi: 10.1074/jbc.M201896200

Lin, C. H., Peng, P. H., Ko, C. Y., Markhart, A. H., and Lin, T. Y. (2012). Characterization of a novel Y2K-type dehydrin VrDhn1 from Vigna radiata. Plant Cell Physiol. 53, 930-942. doi: 10.1093/pcp/pcs040

Liu, H., Yu, C., Li, H., Ouyang, B., Wang, T., Zhang, J., et al. (2015). Overexpression of ShDHN, a dehydrin gene from Solanum habrochaites enhances tolerance to multiple abiotic stresses in tomato. Plant Sci. 231, 198-211. doi: 10.1016/j. plantsci.2014.12.006

Liu, Y., Liang, J., Sun, L., Yang, X., and Li, D. (2016). Group 3 LEA protein, ZmLEA3, is involved in protection from low temperature stress. Front. Plant Sci. 7:1011. doi: 10.3389/fpls.2016.01011

Liu, Y., Wang, L., Xing, X., Sun, L., Pan, J., Kong, X., et al. (2013). ZmLEA3, a multifunctional group 3 LEA protein from maize (Zea mays L.), is involved in biotic and abiotic stresses. Plant Cell Physiol. 54, 944-959. doi: 10.1093/pcp/ pct047

Malik, A. A., Veltri, M., Boddington, K. F., Singh, K. K., and Graether, S. P. (2017). Genome analysis of conserved dehydrin motifs in vascular plants. Front. Plant Sci. 8:709. doi: 10.3389/fpls.2017.00709

Mittler, R. (2002). Oxidative stress, antioxidants and stress tolerance. Trends Plant Sci. 7, 405-410. doi: 10.1016/S1360-1385(02)02312-9

Petersen, J., Eriksson, S. K., Harryson, P., Pierog, S., Colby, T., Bartels, D., et al. (2012). The lysine-rich motif of intrinsically disordered stress protein CDeT11-24 from Craterostigma plantagineum is responsible for phosphatidic acid binding and protection of enzymes from damaging effects caused by desiccation. J. Exp. Bot. 63, 4919-4929. doi: 10.1093/jxb/ers173

Wise, M. J., and Tunnacliffe, A. (2004). POPP the question: what do LEA proteins do? Trends Plant Sci. 9, 13-17. doi: 10.1016/j.tplants.2003.10.012

Xie, C., Zhang, R. X., Qu, Y. T., Miao, Z. Y., Zhang, Y. Q., Shen, X. Y., et al. (2012). Overexpression of MtCAS31 enhances drought tolerance in transgenic Arabidopsis by reducing stomatal density. New Phytol. 195, 124-135. doi: 10.1111/j.1469-8137.2012.04136.x

Yang, W., Zhang, L., Lv, H., Li, H., Zhang, Y., Xu, Y., et al. (2015). The K-segments of wheat dehydrin WZY2 are essential for its protective functions under temperature stress. Front. Plant Sci. 6:406. doi: 10.3389/fpls.2015.00406ref

Conflict of Interest Statement: The authors declare that the research was conducted in the absence of any commercial or financial relationships that could be construed as a potential conflict of interest.

Copyright (C) 2017 Liu, Song, Li, Yang and Li. This is an open-access article distributed under the terms of the Creative Commons Attribution License (CC BY). The use, distribution or reproduction in other forums is permitted, provided the original author(s) or licensor are credited and that the original publication in this journal is cited, in accordance with accepted academic practice. No use, distribution or reproduction is permitted which does not comply with these terms. 